\title{
Tumour cell dormancy as a contributor to the reduced survival of GBM patients who received standard therapy
}

\author{
LUQING TONG $^{1,2,5^{*}}$, LI YI ${ }^{1,2,5^{*}}$, PEIDONG LIU ${ }^{1,2,5^{*}}$, IRUNI ROSHANIE ABEYSEKERA ${ }^{1-3}$, LONG HAI $^{1,2,5}$, \\ TAO LI ${ }^{1,2,5}$, ZHENNAN TAO ${ }^{1,2,5}$, HAIWEN MA ${ }^{1,2,5}$, YANG XIE ${ }^{1,2,5}$, YUBAO HUANG ${ }^{4}$, \\ SHENGPING YU ${ }^{1,2,5}$, JIABO LI ${ }^{1,2,5}$, FENG YUAN ${ }^{1,2,5}$ and XUEJUN YANG ${ }^{1,2,5}$ \\ ${ }^{1}$ Department of Neurosurgery, Tianjin Medical University General Hospital; ${ }^{2}$ Laboratory of Neuro-Oncology, \\ Tianjin Neurological Institute, Tianjin 300052; ${ }^{3}$ Department of Physiology and Pathophysiology, \\ Tianjin Medical University, Tianjin 300070; ${ }^{4}$ Department of Neurosurgery, Tianjin Haihe Hospital, Tianjin 300222; \\ ${ }^{5}$ Key Laboratory of Post-Trauma Neuro-Repair and Regeneration in Central Nervous System, \\ Ministry of Education, Tianjin 300052, P.R. China
}

Received November 10, 2017; Accepted April 19, 2018

DOI: 10.3892/or.2018.6425

\begin{abstract}
Glioblastoma multiforme (GBM) is a fatal cancer with varying life expectancy, even for patients undergoing the same standard therapy. Identification of differentially expressed genes in GBM patients with different survival rates may benefit the development of effective therapeutic strategies. In the present study, key pathways and genes correlated with survival in GBM patients were screened with bioinformatic analysis. Included in the study were 136 eligible patients who had undertaken surgical resection of GBM followed by temozolomide (TMZ) chemoradiation and long-term therapy with TMZ. A total of 383 differentially expressed genes (DEGs) related to GBM survival were identified. Gene Ontology and pathway enrichment analysis as well as hub gene screening and module analysis were performed. As expected, angiogenesis and migration of GBM cells were closely correlated with a poor prognosis. Importantly, the results also indicated that cell dormancy was an essential contributor to the reduced survival of GBM patients. Given the lack of specific targeted genes and pathways known to be involved in tumour cell dormancy, we proposed enriched candidate genes related to the negative regulation of cell proliferation, signalling pathways regulating pluripotency of stem cells and neuroactive ligand-receptor interaction, and 3 hub genes (FTH1, GRM1 and DDIT3). Maintaining persistent cell dormancy or preventing tumour
\end{abstract}

Correspondence to: Professor Xuejun Yang, Department of Neurosurgery, Tianjin Medical University General Hospital, 154 An-shan Road, Heping, Tianjin 300052, P.R. China

E-mail: ydenny@126.com

${ }^{*}$ Contributed equally

Key words: glioblastoma multiforme, standard therapy, survival, dormancy, bioinformatics cells from entering dormancy during chemoradiation should be a promising therapeutic strategy.

\section{Introduction}

Glioblastoma multiforme (GBM), with a 5-year survival rate of $5.1 \%$ (1), has a high fatality rate. The standard therapy of maximal safe resection or subtotal resection followed by concurrent chemoradiation and adjuvant temozolomide (TMZ) is recommended by the Clinical Practice Guidelines for Oncology (2) in the National Comprehensive Cancer Network $^{\circledR}$ (NCCN). This therapeutic strategy is widely carried out all over the world. However, the lifespan of GBM patients varies by relative increments from less than 6 months to more than 3 years. Furthermore, few studies or drugs have been designed to improve the survival time of GBM patients who have undertaken standard therapy.

Cancer cell genotypes, in combination with expression programmes, govern tumour immune fitness, evolution and resistance to therapy (3). In recent years, studies such as those of The Cancer Genome Atlas (TCGA), have charted the genetic landscape and derived from it the bulk expression states of GBM, identifying driver mutations and defining tumour subtypes based on specific transcriptional profiles $(4,5)$. Currently, there is a lack of knowledge concerning the reasons for the variable survival times of GBM patients who have undertaken standard therapy. Therefore, systematic screening of differentially expressed genes (DEGs) and cellular pathways in patients with different survival periods may be of great significance for the development of more effective diagnostic and therapeutic strategies.

In the present study, 136 eligible patients who had undertaken standard therapy were selected from TCGA profiles. DEGs correlating with survival time were identified from 12,042 genes. The most important hub genes and signalling pathways were screened by Gene Ontology (GO) analysis, pathway enrichment analysis, protein-protein interaction (PPI) network and module analysis. In addition, the importance of 
the hub genes was validated by an additional glioma dataset. Finally, evidence in the published literature is discussed to illustrate the importance of these genes and pathways. The recognition of the pivotal genes and pathways that affect the survival of GBM patients who had undertaken standard therapy may be a potential benefit for developing therapeutic strategies.

\section{Materials and methods}

Microarray data. The gene expression (AffyU133a) and phenotypes of the TCGA GBM data were downloaded from UCSC Xena (https://xenabrowser.net/datapages), which is a hub for gene data deposit and retrieval. The gene expression profile was evaluated experimentally using the Affymetrix HT Human Genome U133a microarray platform by the Broad Institute of MIT and Harvard University Cancer Genomic Characterization Center. Level 3 interpreted level data was originated from the TCGA data coordination center. This dataset showed the gene-level transcription estimates and data were in log space. The profile of phenotypes included detailed clinical information corresponding to each of the samples. The profile of Tumor Glioma-French-284-MAS5.0-u133p2 was chosen to validate the importance of the hub genes. It included 8 control samples, 24 grade II glioma samples, 85 grade III glioma samples and 159 grade IV GBM samples. And 272 samples were recorded with their exact survival time. Hub gene expression profiles, historical profiles and survival profiles were downloaded from R2 (http://r2platform.com), a web-based genomic analysis and visualization application.

Sample screening and group assignment. The included samples were from patients who had undertaken surgical resection of the tumor, followed by TMZ chemoradiation and long-term therapy with TMZ. Homogeneity was of great importance for this study, so exclusion criteria were established: i) treated primary GBM; ii) recurrent tumor and normal tissue; iii) Karnofsky performance scores $<60$; iv) no exact survival time; and v) no gene expression files. A total of 136 eligible samples were selected according to the above criteria. General characteristics of samples were analyzed with SPSS software (version 22; IBM Corp., Armonk, NY, USA). Median survival time \pm 10 percentiles was set as a boundary of group assignment. There were 54 samples in each group.

Identification of DEGs. DEGs were analyzed through the online tool Morpheus (https://software.broadinstitute.org/ morpheus/), a versatile matrix visualization and analysis software. Based on the Morpheus analysis, the dataset was adjusted by robust z-score. A total of 383 DEGs were identified through t-test with a P-value cut-off of $\leq 0.05$; of which, 259 were upregulated and 124 were downregulated. P-value cut-off of $\leq 0.01$ was indispensable for hub gene candidates. Both the heat map and dataset of results were downloaded.

Gene Ontology and pathway enrichment analysis of DEGs. Gene Ontology analysis (GO) and KEGG pathway analysis were conducted through DAVID (https://david.ncifcrf.gov/ home.jsp), a database for annotation, visualization and integrated discovery. Upregulated genes and downregulated genes were analyzed respectively and $\mathrm{P} \leq 0.05$ was considered as statistically significant. Annotation tables were downloaded.

Hub gene screening and module analysis. Search tool for the retrieval of interacting genes (STRING) (https://string-db. org/) is a database of prediction of protein-protein interaction (PPI). DEGs were mapped to STRING to conduct PPI analysis, and a minimum required interaction score $\geq 0.4$ was set as significant. A simple tabular text was downloaded. With a differentially expressed $P \leq 0.01$, the most active 11 genes (the last two genes had the same nodes) in PPI networks were considered as hub genes. The relationships of hub genes between expression and glioma histology or survival were analyzed with GraphPad Prism software (version 6). The RNA levels of the 11 hub genes in 136 eligible samples were analyzed with the online tool Morpheus. PPI networks were constructed using the Cytoscape software (version 3.5.1). The modules of PPI networks were filtered with the plug-in Molecular Complex Detection (MCODE) in Cytoscape. MCODE scores $>3$ and number of nodes $>5$ were necessary for modules. Moreover, the function and pathway enrichment analysis were conducted for DEGs in the modules. $\mathrm{P} \leq 0.05$ was considered to be significant.

\section{Results}

Eligible patients. From the inclusion and exclusion criteria described above, 136 eligible patients who had undertaken surgical resection for GBM followed by TMZ chemoradiation and long-term therapy with TMZ were selected. The median survival rate for the patients was 448 days [ $95 \%$ confidence interval (CI), 421 to 475 days]. To obtain more significant differences and to reduce sample capacity loss, patients with survival times that were shorter or longer than the median survival time by \pm 10 percentiles were set as short and long survival groups, respectively. Each group had 54 patients, and differences in age, sex and subtypes were not significant (Table I).

Identification of DEGs. The TCGA gene expression profile identified 12,042 genes. Based on t-test analysis with $\mathrm{P} \leq 0.05$ criterion in Morpheus, a total of 383 genes (259 upregulated and 124 downregulated) were identified after analysis of the two groups. A DEG expression heat map is shown in Fig. 1.

GO term enrichment analysis and KEGG pathway analysis. All DEGs were uploaded to the online software DAVID (https://david.ncifcrf.gov/home.jsp) to identify overrepresented GO categories and KEGG pathways. According to the reverse order of P-values, the top two terms are shown in Tables II and III.

GO analysis results (Table II) showed that the upregulated DEGs were significantly enriched in the biological processes (BP) of angiogenesis and negative regulation of cell proliferation. For molecular function (MF), upregulated DEGs were enriched in hydrolase activity, hydrolysing $O$-glycosyl compounds and integrin binding. Moreover, GO cell component (CC) analysis also showed that the upregulated DEGs were significantly enriched in extracellular exosome and extracellular space. Downregulated DEGs 
Table I. Clinical and subtype features of the GBM sample groups.

\begin{tabular}{|c|c|c|c|}
\hline Variables & Short survival & Long survival & P-value \\
\hline No. of patients & 54 & 54 & \\
\hline Survival, in days & & & $\mathrm{P}<0.001^{\mathrm{a}}$ \\
\hline Median (range) & $250(47-394)$ & $681(502-1977)$ & \\
\hline Age, in years & & & $0.080^{\mathrm{b}}$ \\
\hline Mean (range) & $52.9(18-77)$ & $57.6(17-83)$ & \\
\hline Sex, n $(\%)$ & & & $0.425^{\mathrm{c}}$ \\
\hline Male & $36(66.7)$ & $32(59.3)$ & \\
\hline Female & $18(33.3)$ & $12(40.7)$ & \\
\hline GBM subtype, $\mathrm{n}$ & & & $0.782^{c}$ \\
\hline Classical & 13 & 17 & \\
\hline Mesenchymal & 20 & 16 & \\
\hline Neural & 7 & 8 & \\
\hline Proneural & 14 & 13 & \\
\hline
\end{tabular}

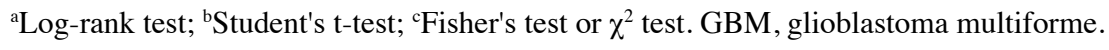

Table II. Gene Ontology analysis of the differentially expressed genes correlated with the survival of the GBM patients.

\begin{tabular}{|c|c|c|c|c|c|}
\hline Expression & Category & Term & Count & $\%$ & P-value \\
\hline \multirow[t]{6}{*}{ Upregulated } & BP & GO:0001525-angiogenesis & 13 & 5.306122 & 7.73E-05 \\
\hline & BP & $\begin{array}{l}\text { GO:0008285-negative regulation } \\
\text { of cell proliferation }\end{array}$ & 16 & 6.530612 & $5.01 \mathrm{E}-04$ \\
\hline & $\mathrm{MF}$ & $\begin{array}{l}\text { GO:0004553-activity, hydrolyzing } \\
\text { O-glycosyl compounds }\end{array}$ & 6 & 2.44898 & 5.59E-05 \\
\hline & MF & GO:0005178-integrin binding & 9 & 3.673469 & $1.16 \mathrm{E}-04$ \\
\hline & $\mathrm{CC}$ & GO:0070062-extracellular exosome & 92 & 37.55102 & $1.54 \mathrm{E}-17$ \\
\hline & $\mathrm{CC}$ & GO:0005615-extracellular space & 43 & 17.55102 & $1.75 \mathrm{E}-07$ \\
\hline \multirow[t]{6}{*}{ Downregulated } & BP & $\begin{array}{l}\text { GO:0000122-negative regulation of transcription } \\
\text { from RNA polymerase II promoter }\end{array}$ & 14 & 12.61261 & $3.39 \mathrm{E}-04$ \\
\hline & $\mathrm{BP}$ & GO:0007268-chemical synaptic transmission & 8 & 7.207207 & $5.78 \mathrm{E}-04$ \\
\hline & MF & GO:0003682-chromatin binding & 9 & 8.108108 & 0.002705 \\
\hline & MF & GO:0046982-protein heterodimerization activity & 8 & 7.207207 & 0.023714 \\
\hline & $\mathrm{CC}$ & GO:0005634-nucleus & 50 & 45.04505 & $1.32 \mathrm{E}-04$ \\
\hline & $\mathrm{CC}$ & GO:0014069-postsynaptic density & 7 & 6.306306 & $6.53 \mathrm{E}-04$ \\
\hline
\end{tabular}

GBM, glioblastoma multiforme; GO, Gene Ontology; BP, biological process; MF, molecular function; CC, cell component.

were involved in the negative regulation of transcription from RNA polymerase II promoter and chemical synaptic transmission for BP. For MF, downregulated DEGs were enriched in chromatin binding, protein heterodimerization activity. For CC, they were enriched in nucleus and postsynaptic density.

KEGG analysis results (Table III) illustrated that the upregulated DEGs were significantly enriched in the pathways of lysosome and extracellular matrix (ECM)-receptor interaction. Downregulated DEGs were enriched in the Rap1 signalling pathway and signalling pathways that regulate the pluripotency of stem cells.
Hub gene screening from the PPI network. With the information from the STRING database, the top 11 hub genes (the last two genes had the same nodes) with the most nodes were screened. These hub genes included the following: serpin family E member 1 (SERPINE1), cathepsin B (CTSB), plasminogen activator, urokinase receptor (PLAUR), galactosidase $\beta 1$ (GLB1), FOS such as 1, AP-1 transcription factor subunit (FOSL1), ferritin heavy chain 1 (FTH1), glutamate metabotropic receptor 1 (GRM1), hexosaminidase subunit $\alpha$ (HEXA), heat shock protein family B member 1 ( $H S P B 1)$, DNA damage inducible transcript 3 (DDIT3), and cation dependent mannose-6-phosphate receptor $(M 6 P R)$. 


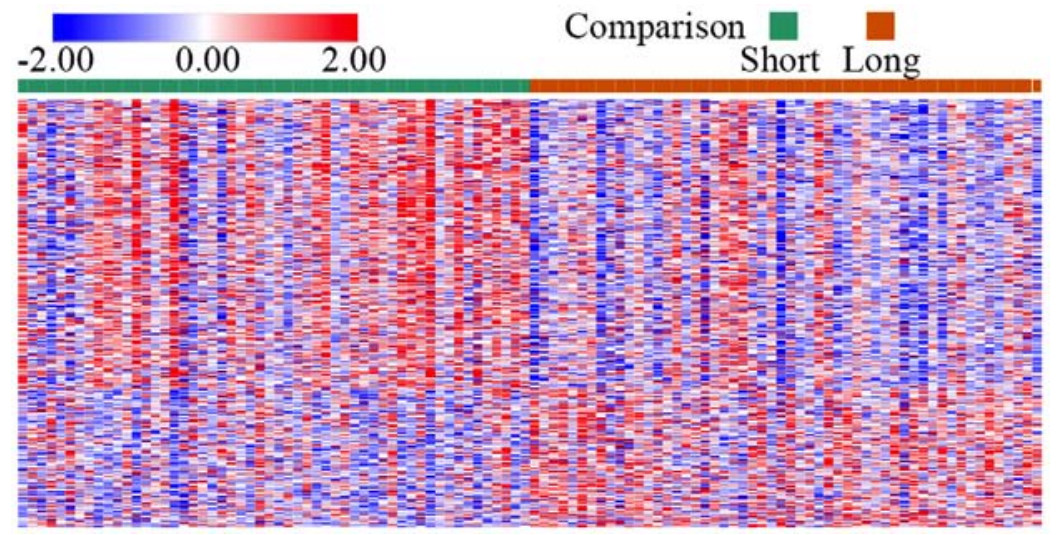

Figure 1. Heat map of 383 differentially expressed genes correlated with the survival of the GBM patients. There were 54 samples in both the short and long survival groups. Based on t-test analysis in Morpheus with a $\mathrm{P} \leq 0.05$ criterion, a total of 383 genes ( 259 upregulated genes and 124 downregulated genes) were identified from 12,042 genes. Red, upregulation; blue, downregulation.
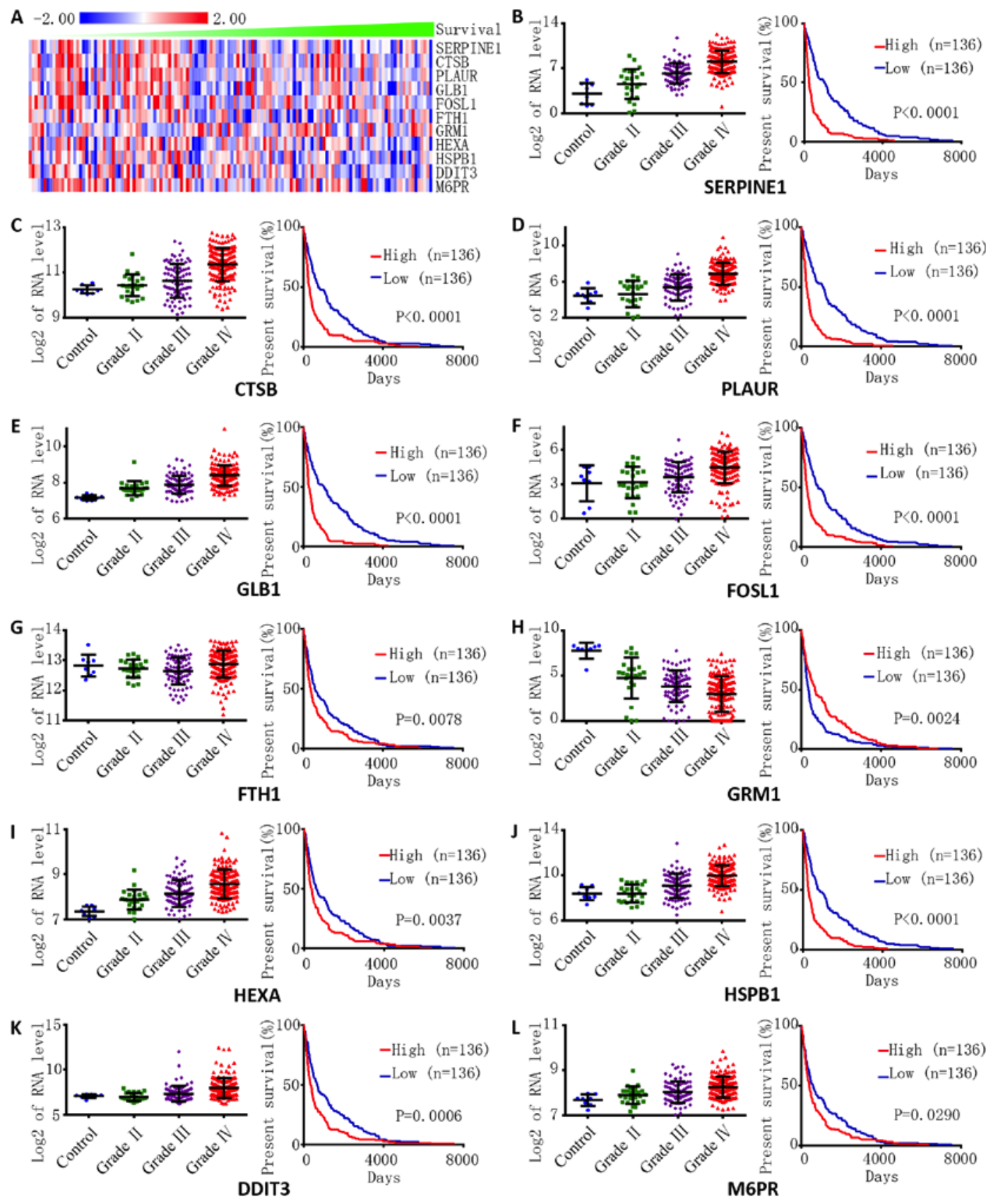

Figure 2. Expression of 11 hub genes. (A) A heat map of 11 hub genes in 136 eligible GBM samples showed that their RNA levels were generally associated with survival. (B-L) The relationship between RNA levels and glioma grade/survival of each hub gene are shown. 
Table III. KEGG pathway analysis of differentially expressed genes associated with the survival of the GBM patients.

\begin{tabular}{|c|c|c|c|c|c|}
\hline Expression & Term & Count & $\%$ & P-value & Genes \\
\hline \multirow[t]{2}{*}{ Upregulated } & hsa04142: Lysosome & 14 & 5.714286 & 7.71E-07 & $\begin{array}{l}\text { GNS, SLC11A1, NPC1, TPP1, PSAP, } \\
\text { ATP6AP1, HEXA, HEXB, CTSD, CTSA } \\
\text { CTSB, M6PR, MANBA, GLB1 }\end{array}$ \\
\hline & $\begin{array}{l}\text { hsa04512: ECM-receptor } \\
\text { interaction }\end{array}$ & 8 & 3.265306 & 0.001753 & $\begin{array}{l}\text { SDC1, LAMB3, LAMA3, ITGA5, } \\
\text { COL6A2, ITGA3, LAMB1, COL5A1 }\end{array}$ \\
\hline \multirow[t]{2}{*}{ Downregulated } & $\begin{array}{l}\text { hsa04015: Rap1 } \\
\text { signaling pathway }\end{array}$ & 6 & 5.405405 & 0.010241 & $\begin{array}{l}\text { P2RY1, SIPA1L3, RAPGEF4, PRKCG, } \\
\text { MAPK11, FGF21 }\end{array}$ \\
\hline & $\begin{array}{l}\text { hsa04550: Signaling } \\
\text { pathways regulating } \\
\text { pluripotency of stem cells }\end{array}$ & 5 & 4.504505 & 0.011701 & NANOG, WNT3, RIF1, ID4, MAPK11 \\
\hline
\end{tabular}

GBM, glioblastoma multiforme; ECM, extracellular matrix.

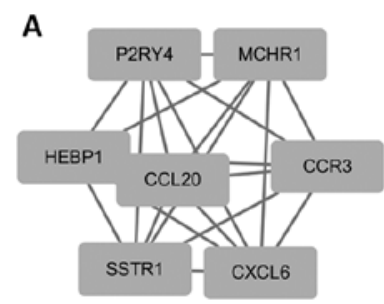

C

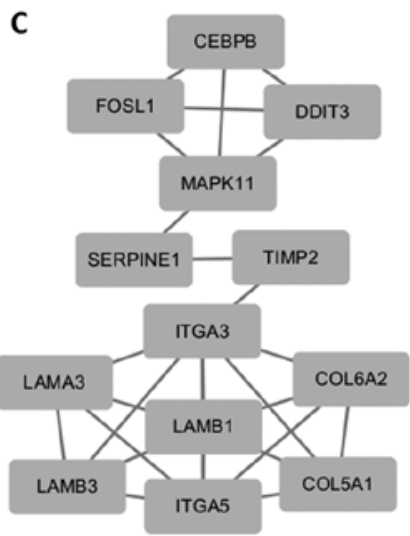

B

\begin{tabular}{lcl}
\hline Term & P-value & Genes \\
\hline $\begin{array}{l}\text { hsa04062:Chemokine } \\
\text { signaling pathway }\end{array}$ & 0.0068 & CCL20, CCR3, CXCL6 \\
$\begin{array}{l}\text { hsa04080:Neuroactive ligand- } \\
\text { receptor interaction }\end{array}$ & 0.0147 & MCHR1, P2RY4, SSTR1 \\
\hline
\end{tabular}

D

\begin{tabular}{lll}
\hline Term & P-value & Genes \\
\hline $\begin{array}{l}\text { hsa04512:ECM-receptor } \\
\text { interaction }\end{array}$ & $1.47 \mathrm{E}-09$ & $\begin{array}{l}\text { LAMB3, LAMA3, ITGA5, COL6A2, } \\
\text { ITGA3, LAMB1, COL5A1 }\end{array}$ \\
hsa04510:Focal adhesion & $2.66 \mathrm{E}-07$ & $\begin{array}{l}\text { LAMB3, LAMA3, ITGA5, COL6A2, } \\
\text { ITGA3, LAMB1, COL5A1 }\end{array}$ \\
\hline
\end{tabular}

Figure 3. Top 2 modules from the protein-protein interaction network. (A and C) The first and second module. (B and D) The enriched pathways of modules $\mathrm{A}$ and $\mathrm{C}$, respectively.

The levels of hub gene expression were closely related to the survival time of 136 eligible GBM patients who had undertaken standard therapy (Fig. 2A). The relationships between hub gene expression and histology and survival were also explored with another dataset (Fig. 2B-L). The expression of FOSL1 was negatively correlated with the grade and survival of glioma (Fig. 2F). The expression of FTH was not significantly related to the grade but positively correlated with survival of glioma (Fig. 2G), and the expression of other genes was positively correlated with the grade and survival of glioma.

Module screening from the PPI network. In addition, a total of 265 nodes and 568 edges were analysed with the Cytoscape plug-in MCODE. The top 2 most significant modules were selected, and the pathway enrichment of the genes involved in these modules was analysed (Fig. 3). Enrichment analysis indicated that the genes in these modules were mainly associated with the chemokine signalling pathway, neuroactive ligand-receptor interactions, ECM-receptor interactions and focal adhesion.

\section{Discussion}

In the present study, the pivotal genes and pathways related to the survival of GBM patients who had undergone standard therapy were explored. Surgical resection of the tumours followed by TMZ chemoradiation and long-term therapy 
with TMZ were required for eligible samples. To eliminate interference from confounding factors, treated tumours, recurrent tumours, normal tissues, and patients with a Karnofsky Performance Status (KPS) $<60$ were excluded. Additionally, the exact survival time was vital for group assignment and gene expression files were essential for further analysis. Finally, 136 eligible patients were included. Their median survival was 448 days (95\% CI, 421-475 days), which was a little longer than the 14.6 months (438 days) reported in a study by Stupp et al (6). This is most likely since all these eligible patients had undergone surgical resection, whereas only $84 \%$ of patients had undergone debulking surgery in the previous study.

Regarding the results of GO pathway analysis, it was significant that the negative regulation of cell proliferation was correlated with the short survival of GBM patients, although unlimited proliferation is one of the essential characteristics of cancers. The process involving growth restraints exerted by ectopic tissue that leads to reversible mitotic arrest is called tumour dormancy (7). The literature published in recent years has revealed a growing focus on tumour cell dormancy, which is probably one of the main reasons for refractory to targeted or conventional therapies (7-10). In glioma, the 'peri-necrotic niche' harbouring HIF- $1 \alpha^{+}$quiescent stem-like cells have been proposed as candidates for long-term tumourigenic cells (11). For the enrichment in angiogenesis of upregulated DEGs, it is widely recognized that the extreme proliferation of new blood vessels in GBM is structurally and functionally abnormal and contributes to a hostile microenvironment (low oxygen tension and high interstitial fluid pressure) that selects for a more malignant phenotype with increasing morbidity and mortality (12-14). In addition, the extracellular space is closely involved with the invasion and migration of glioma cells. It has been reported that a pore space of appropriate size can promote the amoeboid movement of glioma cells (15). Clinically, we also observed that glioma cells spread along the structure of the white matter fibre bundle.

Furthermore, the KEGG pathway analysis showed that the downregulated DEGs were enriched in signalling pathways regulating pluripotency of stem cells. It has been reported that the anti-differentiation strategies of quiescent cells are co-opted by cancer cells (16). Sosa et al reported that downregulated pluripotency is part of tumour cell quiescence and contributes to fuel incurable local or distal recurrences (17). Regarding the enrichment of upregulated DEGs in the lysosome, it is known that the lysosome mediates the process of autophagy and plays multiple context-dependent roles in tumourigenesis and treatment resistance. Previous studies have also shown that the endolysosome is critical for the organization and turnover of epidermal growth factor receptor (EGFR) to maintain tumour growth and invasion (18). For the enrichment of upregulated DEGs in ECM-receptor interaction, glioma cells produce their own ECM environment and use that of the host cells as a bed for migration (19). Integrin directly binds the components of the ECM and provides the traction necessary for cell motility and invasion (20). Regarding the enrichment of downregulated DEGs in the Rap1 signalling pathway, the Ras family of small GTPases is highly expressed in the normal brain, and the Rap1 signalling pathway is necessary to regulate neurite growth and arborization in mammalian neurons $(21,22)$. However, it has been reported that Rap1 signalling also regulates glioma cell motility (23).

PPI networks of DEGs were constructed, and the top-degree hub genes were SERPINE1, CTSB, PLAUR, GLB1, FOSL1, FTH1, GRM1, HEXA, HSPB1, DDIT3 and M6PR. The relationships between RNA expression and histology/survival were also explored with another glioma dataset (Fig. 2). SERPINE1 (also known as PAI-1) encodes a member of the serine proteinase inhibitor (serpin) superfamily. Previous publications have demonstrated that the overexpression of PAI-1 in GBM is significantly correlated with a shorter survival rate (24). Recent research has revealed that the glioma-derived plasminogen activator inhibitor-1 (PAI-1) affects the tumour microenvironment by regulating the recruitment of LRP1-positive mast cells (25). CTSB encodes cathepsin B, a lysosomal cysteine cathepsin. This is consistent with the fact that cathepsin $B$ is a strong predictor of survival in GBM (24). PLAUR (also known as uPAR) encodes the receptor for urokinase plasminogen activator. A correlation between UPAR and invasion of GBM has been repeatedly demonstrated in recent years $(26,27)$. Cathepsin B and uPAR regulate self-renewal of glioma-initiating cells (28). Inhibition of cathepsin B and uPAR inhibits cell invasion in glioma (29) and enhanced radiation-induced apoptosis in gliomainitiating cells (30). GLB1 (also known as EBP) encodes galactosidase $\beta 1$. When bound to elastin-derived peptides, EBP allows GBM cells to adhere to the newly synthesized matrix and increases their aggressiveness (31). Generally, the molecular mechanisms underlying GLB1 in GBM have not been well clarified. FOSL1 (also known as FRA1) encodes the AP-1 transcription factor Fra-1. Only a few literature reports have proposed that Fra-1 takes part in migratory behaviour of GBM cells (32). However, FRA1 expression is associated with the migration of breast cancers (33), and the depletion of FRA1 results in a mesenchymal-epithelial transition (34). FTH1 encodes ferritin heavy chain 1, the major intracellular iron storage protein in cells. Ferritin protects DNA from iron-induced oxidative damage (35), and silencing the ferritin heavy chain can effectively sensitize tumours to chemotherapy in glioma (36). However, the ferritin heavy chain has also been reported to be a negative regulator of ovarian cancer stem cell expansion and epithelial-to-mesenchymal transition (37). GRM1 encodes glutamate metabotropic receptor 1 . Ligand binding to this protein activates a phosphatidylinositol-calcium second messenger system. The Human Protein Atlas (http://www.proteinatlas.org) shows that GRM1 is highly expressed in normal brain tissue and less frequently expressed in glioma tissue and cells. This is consistent with the negative relationship between GRM1 expression and glioma grade (Fig. 2). However, it has also been demonstrated that mGluR1 inhibition induces cell cycle arrest, caspase-dependent apoptosis, and prevents invasion and migration in glioma (38). HEXA encodes a preproprotein that is proteolytically processed to generate the $\alpha$ subunit of the lysosomal enzyme $\beta$-hexosaminidase. In the last century, $\beta$-hexosaminidase was found to be overexpressed in colonic carcinoma (39), ovarian adenocarcinoma (40) and lung cancer (41), but no further research has been conducted to clarify its underlying mechanisms, which need to be further verified. HSPB1 (also known as HP27) encodes heat shock 
protein family B member 1, which functions as a molecular chaperone, probably maintaining denatured proteins in a folding-competent state. Recently, HSPB1 was proposed to be a discriminating short protein that is a long survival factor in GBM (42). HSP27 mediates SPARC-induced changes in glioma morphology and invasion (43), and inhibition of HSP27 alone or in combination with pAKT inhibition may be an effective therapeutic approach (44). DDIT3 (also known as CHOP) encodes DNA damage inducible transcript 3 , which functions as an inhibitor by forming heterodimers with other C/EBP members. In addition, it blocks their DNA binding activity. $\mathrm{CHOP}$ facilitates autophagy and contributes to resistance to treatment in glioma $(45,46)$. Overexpression of CHOP enables immune inhibitory activity of tumourinfiltrating myeloid-derived suppressor cells (MDSCs), which promotes cancer development (47). M6PR encodes mannose6-phosphate receptor, which is related to transportation of phosphorylated lysosomal enzymes. There have been few research efforts involved in determining the influence of M6PR on cancers. Selective M6PR downregulation has a critical role in $\mathrm{CD}^{+} \mathrm{T}$ cell survival, which provides protection against cancer (48). M6PR mediates TMEPAI transport from the Golgi directly into the endo-lysosomal pathway and then indirectly boosts tumourigenesis in lung cancer (49).

Module analysis of the PPI network revealed that the DEGs related to survival were mainly associated with cell migration. GBM secretes chemokines that can promote tumour growth and progression or induce stromal cells to provide a hotbed for tumour growth (50). Neuroactive ligand-receptor interactions have been reported as having a negative effect $(51,52)$ on glioma cell proliferation. It was a foregone conclusion that ECM-receptor interaction and focal adhesion contribute to a poor prognosis, as both are closely related to cancer cell invasion.

It must be acknowledged that microarray analysis has limitations. First, it can only report the level of mRNA expression, usually comparing tumour tissues with non-tumour tissues, and the genes that are highly expressed in tumour tissue are determined by statistical analysis. However, this method cannot analyse gene modification or detect protein function. For example, NLGN3 is a functional protein expressed in normal brain tissue, but its associated normal functional activity promotes cancer growth (53). On the other hand, due to statistical analysis, bioinformatic analysis is not applicable to all cases and samples. For example, the trend in gene expression profiles in each sample is not consistent in Fig. 1, but the trend in most samples is consistent. This is due to small probability events that are almost impossible in a single experiment but inevitable in repeated tests. This indicates that bioinformatic analysis is only an analytical method. Scientific research cannot be entirely dependent on such a method, and the hypotheses yielded by bioinformatic approaches must be demonstrated by various experimental methods and finally proven in clinical practice.

The present study identified key pathways and genes correlated with survival of GBM patients who had undertaken standard therapy. As expected, the angiogenesis and migration of GBM cells contributed to a poor prognosis. Notably, the upregulated genes were enriched in negative regulation of cell proliferation, and the downregulated genes were enriched in signalling pathways regulating pluripotency of stem cells. A total of 3 hub genes (FTH1, GRM1 and DDIT3) had negative effects on cancer cell proliferation, and the module genes of the PPI network were enriched in neuroactive ligand-receptor interaction, which has been reported to have a negative effect on glioma cell proliferation. All the above results highlight that cell dormancy is an essential contributor to the shorter survival of GBM patients who have undertaken standard therapy. The change in the dormant state is probably determined through a process of epigenetic regulation (54). Presumably, the expression and functional level of the dormant genes would be reduced in recurrent tumours. However, as little research has been published concerning this issue, further experimental validation is required to confirm the pathogenesis and mechanisms. Modulating persistent cell dormancy or preventing tumour cells from translating into quiescence during radiochemotherapy should be helpful to prolong the survival of GBM patients under standard treatment.

\section{Acknowledgements}

Not applicable.

\section{Funding}

The present study was supported by the National Natural Science Foundation of China (no. 81472352) and the Tianjin Research Program of Application Foundation and Advanced Technology (no. 15JCZDJC36200).

\section{Availability of data and materials}

The datasets used during the present study are freely available from the corresponding websites.

\section{Authors' contributions}

LT and XY conceived and designed the study. LY, PL, LH and TL downloaded the datasets, selected eligible samples, assigned groups and screened differentially expressed genes (DEGs). They also analyzed the differences of age, sex and subtypes of the groups. ZT and HM underwent Gene Ontology and pathway enrichment analysis of DEGs. YX, YH and SY conducted PPI analysis and selected hub genes. In addition, they analyzed the relationships of hub genes between expression and glioma histology or survival with another glioma dataset. JL and FY conducted module analysis. LT, LY and PL reviewed many relevant articles and analyzed the research progress of related pathways and genes. LT, XY and IRA wrote the paper. LT, LY, PL and IRA reviewed and edited the manuscript. All authors read and approved the manuscript and agree to be accountable for all aspects of the research in ensuring that the accuracy or integrity of any part of the work are appropriately investigated and resolved.

\section{Ethics approval and consent to participate}

No procedures performed in the study involved human participants. 


\section{Consent for publication}

Not applicable.

\section{Competing interests}

The authors state that they have no competing interests.

\section{References}

1. Ostrom QT, Gittleman H, Fulop J, Liu M, Blanda R, Kromer C, Wolinsky Y, Kruchko C AND Barnholtz-Sloan JS: CBTRUS Statistical Report: Primary Brain and Central Nervous System Tumors Diagnosed in the United States in 2008-2012. Neuro Oncol 17 (Suppl 4): iv1-iv62, 2015.

2. Nabors LB, Portnow J, Ammirati M, Baehring J, Brem H, Brown P, Butowski N, Chamberlain MC, Fenstermaker RA, Friedman A et al: Central Nervous System Cancers, Version 1.2015. J Natl Compr Canc Netw 13: 1191-1202, 2015.

3. Venteicher AS, Tirosh I, Hebert C, Yizhak K, Neftel C, Filbin MG, Hovestadt V, Escalante LE, Shaw ML, Rodman C, et al: Decoupling genetics, lineages, and microenvironment in IDH-mutant gliomas by single-cell RNA-seq. Science 355: 355 , 2017.

4. Brennan CW, Verhaak RG, McKenna A,Campos B, NoushmehrH, Salama SR, Zheng S, Chakravarty D, Sanborn JZ, Berman SH, et al; TCGA Research Network: The somatic genomic landscape of glioblastoma. Cell 155: 462-477, 2013

5. Verhaak RG, Hoadley KA, Purdom E, Wang V, Qi Y, Wilkerson MD, Miller CR, Ding L, Golub T, Mesirov JP, et al; Cancer Genome Atlas Research Network: Integrated genomic analysis identifies clinically relevant subtypes of glioblastoma characterized by abnormalities in PDGFRA, IDHI, EGFR, and NF1. Cancer Cell 17: 98-110, 2010.

6. Stupp R, Mason WP, van den Bent MJ, Weller M, Fisher B, Taphoorn MJ, Belanger K, Brandes AA, Marosi C, Bogdahn U, et al; European Organisation for Research and Treatment of Cancer Brain Tumor and Radiotherapy Groups; National Cancer Institute of Canada Clinical Trials Group: Radiotherapy plus concomitant and adjuvant temozolomide for glioblastoma. $\mathrm{N}$ Engl J Med 352: 987-996, 2005.

7. Klein CA: Framework models of tumor dormancy from patientderived observations. Curr Opin Genet Dev 21: 42-49, 2011.

8. Goss PE and Chambers AF: Does tumour dormancy offer a therapeutic target? Nat Rev Cancer 10: 871-877, 2010.

9. Aguirre-Ghiso JA: Models, mechanisms and clinical evidence for cancer dormancy. Nat Rev Cancer 7: 834-846, 2007.

10. Sosa MS, Bragado P and Aguirre-Ghiso JA: Mechanisms of disseminated cancer cell dormancy: An awakening field. Nat Rev Cancer 14: 611-622, 2014.

11. Ishii A, Kimura T, Sadahiro H, Kawano H, Takubo K, Suzuki M and Ikeda E: Histological characterization of the tumorigenic 'peri-necrotic niche' harboring quiescent stem-like tumor cells in glioblastoma. PLoS One 11: e0147366, 2016.

12. Jain RK, di Tomaso E, Duda DG, Loeffler JS, Sorensen AG and Batchelor TT: Angiogenesis in brain tumours. Nat Rev Neurosci 8: 610-622, 2007.

13. Tate MC and Aghi MK: Biology of angiogenesis and invasion in glioma. Neurotherapeutics 6: 447-457, 2009.

14. Jhaveri N, Chen TC and Hofman FM: Tumor vasculature and glioma stem cells: Contributions to glioma progression. Cancer Lett 380: 545-551, 2016

15. Huang Y, Tong L, Yi L, Zhang C, Hai L, Li T, Yu S, Wang W, Tao Z, Ma H, et al: Three-dimensional hydrogel is suitable for targeted investigation of amoeboid migration of glioma cells. Mol Med Rep 17: 250-256, 2018.

16. Sang L, Roberts JM and Coller HA: Hijacking HES1: How tumors co-opt the anti-differentiation strategies of quiescent cells. Trends Mol Med 16: 17-26, 2010.

17. Sosa MS, Parikh F, Maia AG, Estrada Y, Bosch A, Bragado P, Ekpin E, George A, Zheng Y, Lam HM, et al: NR2F1 controls tumour cell dormancy via SOX9- and RAR $\beta$-driven quiescence programmes. Nat Commun 6: 6170, 2015.

18. Kondapalli KC, Llongueras JP, Capilla-González V, Prasad H, Hack A, Smith C, Guerrero-Cázares H, Quiñones-Hinojosa A and Rao R: A leak pathway for luminal protons in endosomes drives oncogenic signalling in glioblastoma. Nat Commun 6 : $6289,2015$.
19. Noreen R, Moenner M, Hwu Y and Petibois C: FTIR spectroimaging of collagens for characterization and grading of gliomas. Biotechnol Adv 30: 1432-1446, 2012.

20. Desgrosellier JS and Cheresh DA: Integrins in cancer: Biological implications and therapeutic opportunities. Nat Rev Cancer 10: 9-22, 2010.

21. Kawabe H, Neeb A, Dimova K, Young SM Jr, Takeda M, Katsurabayashi S, Mitkovski M, Malakhova OA, Zhang DE, Umikawa M, et al: Regulation of Rap2A by the ubiquitin ligase Nedd4-1 controls neurite development. Neuron 65: 358-372, 2010.

22. Schwamborn JC and Püschel AW: The sequential activity of the GTPases Rap1B and Cdc42 determines neuronal polarity. Nat Neurosci 7: 923-929, 2004.

23. Barrett A, Evans IM, Frolov A, Britton G, Pellet-Many C, Yamaji M, Mehta V, Bandopadhyay R, Li N, Brandner S, et al: A crucial role for DOK1 in PDGF-BB-stimulated glioma cell invasion through p130Cas and Rap1 signalling. J Cell Sci 127: 2647-2658, 2014.

24. Colin C, Voutsinos-Porche B, Nanni I, Fina F, Metellus P, Intagliata D, Baeza N, Bouvier C, Delfino C, Loundou A, et al: High expression of cathepsin $\mathrm{B}$ and plasminogen activator inhibitor type- 1 are strong predictors of survival in glioblastomas. Acta Neuropathol 118: 745-754, 2009.

25. Roy A, Coum A, Marinescu VD, Põlajeva J, Smits A, Nelander S, Uhrbom L, Westermark B, Forsberg-Nilsson K, Pontén F, et al: Glioma-derived plasminogen activator inhibitor-1 (PAI-1) regulates the recruitment of LRP1 positive mast cells. Oncotarget 6: 23647-23661, 2015.

26. Hu J, Muller KA, Furnari FB, Cavenee WK, VandenBerg SR and Gonias SL: Neutralizing the EGF receptor in glioblastoma cells stimulates cell migration by activating uPAR-initiated cell signaling. Oncogene 34: 4078-4088, 2015.

27. Raghu H, Gondi CS, Dinh DH, Gujrati M and Rao JS: Specific knockdown of uPA/uPAR attenuates invasion in glioblastoma cells and xenografts by inhibition of cleavage and trafficking of Notch-1 receptor. Mol Cancer 10: 130, 2011.

28. Gopinath S, Malla R, Alapati K, Gorantla B, Gujrati M, Dinh DH and Rao JS: Cathepsin B and uPAR regulate self-renewal of glioma-initiating cells through GLI-regulated Sox2 and Bmil expression. Carcinogenesis 34: 550-559, 2013.

29. Rao Malla R, Gopinath S, Alapati K, Gorantla B, Gondi CS and Rao JS: Knockdown of cathepsin B and uPAR inhibits CD151 and $\alpha 3 \beta 1$ integrin-mediated cell adhesion and invasion in glioma. Mol Carcinog 52: 777-790, 2013.

30. Malla RR, Gopinath S, Alapati K, Gorantla B, Gondi CS and Rao JS: uPAR and cathepsin B inhibition enhanced radiationinduced apoptosis in gliomainitiating cells. Neuro Oncol 14: 745-760, 2012

31. Coquerel B, Poyer F, Torossian F, Dulong V, Bellon G, Dubus I, Reber A and Vannier JP: Elastin-derived peptides: Matrikines critical for glioblastoma cell aggressiveness in a 3-D system. Glia 57: 1716-1726, 2009.

32. Debinski W and Gibo DM: Fos-related antigen 1 (Fra-1) pairing with and transactivation of JunB in GBM cells. Cancer Biol Ther 11: 254-262, 2011.

33. Belguise K, Cherradi S, Sarr A, Boissière F, Boulle N, SimonyLafontaine J, Choesmel-Cadamuro V, Wang X and Chalbos D: PKC $\theta$-induced phosphorylations control the ability of Fra-1 to stimulate gene expression and cancer cell migration. Cancer Lett 385: 97-107, 2017.

34. Tam WL, Lu H, Buikhuisen J, Soh BS, Lim E, Reinhardt F, Wu ZJ, Krall JA, Bierie B, Guo W, et al: Protein kinase C $\alpha$ is a central signaling node and therapeutic target for breast cancer stem cells. Cancer Cell 24: 347-364, 2013.

35. Thompson KJ, Fried MG, Ye Z, Boyer P and Connor JR: Regulation, mechanisms and proposed function of ferritin translocation to cell nuclei. J Cell Sci 115: 2165-2177, 2002.

36. Liu X, Madhankumar AB, Slagle-Webb B, Sheehan JM, Surguladze N and Connor JR: Heavy chain ferritin siRNA delivered by cationic liposomes increases sensitivity of cancer cells to chemotherapeutic agents. Cancer Res 71: 2240-2249, 2011.

37. Lobello N, Biamonte F, Pisanu ME, Faniello MC, Jakopin Ž Chiarella E, Giovannone ED, Mancini R, Ciliberto G, Cuda G, et al: Ferritin heavy chain is a negative regulator of ovarian cancer stem cell expansion and epithelial to mesenchymal transition. Oncotarget 7: 62019-62033, 2016. 
38. Zhang C, Yuan XR, Li HY, Zhao ZJ, Liao YW, Wang XY, Su J, Sang SS and Liu Q: Anti-cancer effect of metabotropic glutamate receptor 1 inhibition in human glioma U87 cells: Involvement of PI3K/Akt/mTOR pathway. Cell Physiol Biochem 35: 419-432, 2015.

39. Brattain MG, Green C, Kimball PM, Marks M and Khaled M Isoenzymes of beta-hexosaminidase from normal rat colon and colonic carcinoma. Cancer Res 39: 4083-4090, 1979.

40. Chatterjee SK, Chowdhury K, Bhattacharya M and Barlow JJ Beta-hexosaminidase activities and isoenzymes in normal human ovary and ovarian adenocarcinoma. Cancer 49: 128-135, 1982.

41. Narita M, Taniguchi N, Makita A, Kodama T, Araki E and Oikawa K: Elevated activity of beta-hexosaminidase and sulfhydryl modification in the B-variant of human lung cancer. Cancer Res 43: 5037-5042, 1983.

42. Gimenez M, Marie SK, Oba-Shinjo S, Uno M, Izumi C, Oliveira JB and Rosa JC: Quantitative proteomic analysis shows differentially expressed HSPB 1 in glioblastoma as a discriminating short from long survival factor and NOVA1 as a differentiation factor between low-grade astrocytoma and oligodendroglioma. BMC Cancer 15: 481, 2015.

43. Golembieski WA, Thomas SL, Schultz CR, Yunker CK, McClung HM, Lemke N, Cazacu S, Barker T, Sage EH, Brodie C, et al: HSP27 mediates SPARC-induced changes in glioma morphology, migration, and invasion. Glia 56: 1061-1075, 2008.

44. Schultz CR, Golembieski WA, King DA, Brown SL, Brodie C and Rempel SA: Inhibition of HSP27 alone or in combination with pAKT inhibition as therapeutic approaches to target SPARC-induced glioma cell survival. Mol Cancer 11: 20, 2012.

45. Rouschop KM, van den Beucken T, Dubois L, Niessen H, Bussink J, Savelkouls K, Keulers T, Mujcic H, Landuyt W, Voncken JW, et al: The unfolded protein response protects human tumor cells during hypoxia through regulation of the autophagy genes MAPILC3B and ATG5. J Clin Invest 120: 127-141, 2010

46. Liu WT, Huang CY, Lu IC and Gean PW: Inhibition of glioma growth by minocycline is mediated through endoplasmic reticulum stress-induced apoptosis and autophagic cell death. Neuro Oncol 15: 1127-1141, 2013.
47. Thevenot PT, Sierra RA, Raber PL, Al-Khami AA, TrilloTinoco J, Zarreii P, Ochoa AC, Cui Y, Del Valle L and Rodriguez PC: The stress-response sensor chop regulates the function and accumulation of myeloid-derived suppressor cells in tumors. Immunity 41: 389-401, 2014.

48. Ahmed KA, Wang L, Griebel P, Mousseau DD and Xiang J: Differential expression of mannose-6-phosphate receptor regulates T cell contraction. J Leukoc Biol 98: 313-318, 2015.

49. Luo S, Jing L, Zhao T, Li Y, Liu Z and Diao A: Ubiquitination and dynactin regulate TMEPAI lysosomal trafficking. Sci Rep 7: 42668, 2017.

50. Somasundaram R and Herlyn D: Chemokines and the microenvironment in neuroectodermal tumor-host interaction. Semin Cancer Biol 19: 92-96, 2009.

51. Barbieri F, Pattarozzi A, Gatti M, Aiello C, Quintero A Lunardi G, Bajetto A, Ferrari A, Culler MD and Florio T: Differential efficacy of SSTR1, -2 , and -5 agonists in the inhibition of C6 glioma growth in nude mice. Am J Physiol Endocrinol Metab 297: E1078-E1088, 2009.

52. Barbieri F, Pattarozzi A, Gatti M, Porcile C, Bajetto A, Ferrari A, Culler MD and Florio T: Somatostatin receptors 1, 2, and 5 cooperate in the somatostatin inhibition of C6 glioma cell proliferation in vitro via a phosphotyrosine phosphatase-etadependent inhibition of extracellularly regulated kinase-1/2. Endocrinology 149: 4736-4746, 2008.

53. Venkatesh HS, Johung TB, Caretti V, Noll A, Tang Y, Nagaraja S, Gibson EM, Mount CW, Polepalli J, Mitra SS, et al: Neuronal Activity Promotes Glioma Growth through Neuroligin-3 Secretion. Cell 161: 803-816, 2015.

54. Lyu T, Jia N, Wang J, Yan X, Yu Y, Lu Z, Bast RC Jr, Hua K and Feng W: Expression and epigenetic regulation of angiogenesis-related factors during dormancy and recurrent growth of ovarian carcinoma. Epigenetics 8: 1330-1346, 2013. 